\title{
Irreversible Dynamics of Infinite Fermion Systems
}

\author{
E. B. Davies \\ St. John's College, Oxford, OX1 3JP, England
}

\begin{abstract}
We investigate the irreversible dynamics of infinite systems as specified by completely positive, strongly continuous, one-parameter semigroups on a suitable $C^{*}$-algebra. Having shown how to construct such a semigroup from a fairly general evolution equation we determine when the semigroup is spatial with respect to a given representation of the algebra. A special class of exactly soluble evolution equations on the CAR algebra is studied in detail in order to test conjectured extensions of the theory.
\end{abstract}

\section{$\S 1$. Introduction}

If $\mathscr{A}$ is a $C^{*}$-algebra, which we shall always assume possesses an identity element 1 , a dynamical semigroup on $\mathscr{A}$ is defined as a strongly continuous one-parameter semigroup of completely positive $\left[12\right.$, p. 136; 47] identity-preserving maps $T_{t}$ of $\mathscr{A}$ into itself. Such semi-groups arise in various contexts in non-equilibrium quantum statistical mechanics $[12,27]$, sometimes in the Heisenberg picture as above, and sometimes in the Schrodinger picture. They may be obtained in the weak or singular coupling limit when a system interacts with an infinite external reservoir $[8,13,23$, $35,38]$. In the converse direction given a dynamical semigroup one may frequently dilate it to a dynamical group, specified in some sense by a Hamiltonian, on a larger system $[11,20,22,33]$.

Two special types of dynamical semigroup are fairly well understood. In the first case [12], $\mathscr{A}$ is the algebra $\mathscr{L}(\mathscr{H})$ of all bounded operators on a separable Hilbert space $\mathscr{H}$ and $T_{t}$ is obtained by duality from a one-parameter semigroup of the space of trace class operators on $\mathscr{H}$. In the second case $[19,30,42], T_{t}$ is a one-parameter group of *-automorphisms of $\mathscr{A}$. Results known in one or other of these two special cases motivate much of the present work.

Associated with a dynamical semigroup $T_{t}$ on $\mathscr{A}$ is its evolution equation

$$
\frac{d X}{d t}=Z(X)
$$


where the infinitesimal generator $Z$ of the semigroup is a closed densely defined operator on $\mathscr{A}$. Also associated with $T_{t}$ is the one-parameter semigroup $T_{t}^{*}$ which maps the compact convex set $\mathscr{S}$ of states of $\mathscr{A}$ into itself. In this paper we construct and investigate the properties of certain dynamical semigroups whose evolution equations are of a form previously studied in $[9,10]$. Two particular goals are to investigate the stability and spatial implementation of temporally invariant states, defined as elements of

$$
\mathscr{S}_{T}=\left\{\phi \in \mathscr{S}: T_{t}^{*} \phi=\phi \text { for all } t \geqq 0\right\} .
$$

In most of the paper we assume for definiteness that $\mathscr{A}$ is the CAR algebra. Much of the analysis could, however, be carried through for the CCR algebra, a quantum spin system or even a general $C^{*}$-algebra with quasi-local structure. We leave such modifications to the reader. We conclude this section by recalling some standard results about the CAR algebra [45] and fixing our notation.

The CAR algebra $\mathscr{A}$ over a complex Hilbert space $\mathscr{H}$ is a simple $C^{*}$-algebra generated by bounded operators $a(f)$ which are conjugate linear with respect to $f \in \mathscr{H}$ and satisfy

$$
\begin{aligned}
& a(f) a^{*}(g)+a^{*}(g) a(f)=<g, f>1 \\
& a^{*}(f) a^{*}(g)+a^{*}(g) a^{*}(f)=0
\end{aligned}
$$

for all $f, g \in \mathscr{H}$. The symbol $a^{\#}(f)$ denotes $a^{*}(f)$ or $a(f)$ indifferently. By a Wick monomial of degree $n$ we mean a product of $n$ operators $a^{\#}\left(f^{r}\right)$. We say the monomial is normal (anti-normal) ordered if the $a^{*}$ 's are all to the left (resp. right) of all the $a$ 's.

For every unitary map $U$ on $\mathscr{H}$ there is a ${ }^{*}$-automorphism $\alpha(U)$ of $\mathscr{A}$ such that

$$
\alpha(U)\left\{a^{\#}(f)\right\}=a^{\#}(U f)
$$

for all $f \in \mathscr{H}$. In particular there is an automorphism $\theta$ of $\mathscr{A}$ such that $\theta^{2}=1$ and

$$
\theta\left\{a^{\#}(f)\right\}=-a^{\#}(f)
$$

for all $f \in \mathscr{H}$. We define

$$
\begin{aligned}
& \mathscr{A}_{0}=\{X \in \mathscr{A}: \theta(X)=X\} \\
& \mathscr{A}_{1}=\{X \in \mathscr{A}: \theta(X)=-X\} .
\end{aligned}
$$

The observable $C^{*}$-subalgebra $\mathscr{A}_{0}$ of $\mathscr{A}$ is known to be simple and even $*_{\text {-iso- }}$ morphic to $\mathscr{A}[17 ; 19$, p. $306 ; 48]$. by

If $\mathscr{H}=L^{2}\left(\mathbb{R}^{3}\right), f \in \mathscr{H}$ and $x \in \mathbb{R}^{3}$, we define $f_{x} \in \mathscr{H}$ and the unitary map $U_{x}$ on $\mathscr{H}$

$$
f_{x}(y)=\left(U_{x} f\right)(y)=f(y-x) .
$$

If $B \in \mathscr{A}$ we define $B_{x} \in \mathscr{A}$ and the automorphism $\alpha_{x}$ of $\mathscr{A}$ by

$$
B_{x}=\alpha_{x}(B)=\alpha\left(U_{x}\right) B .
$$

We shall call various maps spatially homogeneous if they are invariant under all space translations. 
If $S$ is an unbounded spatially homogeneous self-adjoint operator on $\mathscr{H}=L^{2}\left(\mathbb{R}^{3}\right)$ we define $f_{t} \in \mathscr{H}$ by

$$
f_{t}=e^{i S t} f \text {. }
$$

If $B \in \mathscr{A}$ we define $B_{t} \in \mathscr{A}$ and the automorphism $S_{t}$ of $\mathscr{A}$ by

$$
B_{t}=S_{t}(B)=\alpha\left(e^{i S t}\right) B .
$$

The infinitesimal generator of the group $S_{t}$ is an unbounded derivation $[6,36]$ on $\mathscr{A}$ which we denote by $D_{S}$. In the symbol $f_{x t}$ or $B_{x t}$ the first index will always refer to the space translation and the second to the time translation.

\section{§2. A Spatially Homogeneous Model}

We let $\mathscr{A}$ be the CAR algebra over $\mathscr{H}=L^{2}\left(\mathbb{R}^{3}\right)$ and consider the formal evolution equation

$$
\frac{d X}{d t}=D(X)+\int_{\mathbb{R}^{3}}\left\{2 B_{x}^{*} \theta(X) B_{x}-B_{x}^{*} B_{x} X-X B_{x}^{*} B_{x}\right\} d^{3} x
$$

where $D$ is the derivation associated to the single-particle Hamiltonian $S=-\frac{1}{2} \Delta$ and $B$ is a Wick monomial of odd degree. This equation is similar to, but more general than, those studied in $[9,10]$. The integral describes a stochastic influence on the fermion system due to its interaction with an infinite external reservoir. The slightly unusual presence of $\theta$ in Equation (2.1) is necessitated by the form of the anticommutation relations. $\theta$ is omitted if $B$ is a Wick monomial of even degree, or if the evolution equation is interpreted as acting on the observable algebra $\mathscr{A}_{0}$.

For all finite $a$ the evolution equation

$$
\frac{d X}{d t}=D(X)+\int_{|x| \leqq a}\left\{2 B_{x}^{*} \theta(X) B_{x}-B_{x}^{*} B_{x} X-X B_{x}^{*} B_{x}\right\} d^{3} x
$$

on $\mathscr{A}$ is soluble because the RHS of Equation (2.2) defines a bounded perturbation of $D$. The solution

$$
X(t)=T_{a}(t) X
$$

defines a dynamical semigroup $T_{a}(t)$ on $\mathscr{A}$ by use of the Trotter product formula as in [12 p. 83]. Following [49], we show that as $a \rightarrow \infty, T_{a}(t)$ converges to a dynamical semigroup $T(t)$ on $\mathscr{A}$. The following theorem may be adapted to quantum spin systems or quasi-local $C^{*}$-algebras by following [40] or [50] respectively. One may also extend it to the case where the interaction term of Equation (2.1) is replaced by a finite sum of similar terms.

\section{Theorem 2.1. Let}

$$
B=a^{\#}\left(f^{1}\right) \ldots a^{\#}\left(f^{n}\right)
$$

where $n$ is odd and each $f^{i}$ lies in Schwartz space $\mathscr{S}$.

Then for all $X \in \mathscr{A}$

$$
\lim _{a \rightarrow \infty} T_{a}(t) X=T(t) X
$$


in norm, uniformly for $t$ in any finite interval. The limit $T(t)$ is a spatially homogeneous dynamical semigroup on $\mathscr{A}$.

Proof. We define the bounded map $J_{x t}: \mathscr{A} \rightarrow \mathscr{A}$ by

$$
\begin{aligned}
J_{x t}(X)= & 2 B_{x,-t}^{*} \theta(X) B_{x,-t} \\
& -B_{x,-t}^{*} B_{x,-t} X-X B_{x,-t}^{*} B_{x,-t} .
\end{aligned}
$$

The solution of

$$
\frac{d X}{d t}=D(X)+\int_{|x| \leqq a} J_{x}(X) d^{3} x
$$

is

$$
\begin{aligned}
T_{a}(t) X= & S_{t} X+\int_{s=0}^{t} \int_{|x| \leqq a} S_{t-s} J_{x} S_{s} X d x d s \\
& +\int_{s=0}^{t} \int_{u=0}^{s} \int_{|x| \leqq a} \int_{|y| \leqq a} S_{t-s} J_{x} S_{s-u} J_{y} S_{u} X d y d x d u d s+\ldots
\end{aligned}
$$

Therefore

$$
\begin{aligned}
S_{-t} T_{a}(t) X= & X+\int_{s=0}^{t} \int_{|x| \leqq a} J_{x s} X d x d s \\
& +\int_{s=0}^{t} \int_{u=0}^{s} \int_{|x| \leqq a} \int_{|y| \leqq a} J_{x s} J_{y u} X d x d y d u d s+\ldots
\end{aligned}
$$

We compare this as $a \rightarrow \infty$ with the expression

$$
\begin{aligned}
S_{-t} T(t) X= & X+\int_{s=0}^{t} \int_{x \in \mathbb{R}^{3}} J_{x s} X d x d s \\
& +\int_{s=0}^{t} \int_{u=0}^{s} \int_{x \in \mathbb{R}^{3}} \int_{y \in \mathbb{R}^{3}} J_{x s} J_{y u} X d x d y d u d s+\ldots
\end{aligned}
$$

whose convergence has to be demonstrated.

Assuming $X$ has the form

$$
X=a^{\#}\left(g^{1}\right) \ldots a^{\#}\left(g^{m}\right)
$$

where each $g^{j}$ lies in $\mathscr{S}$, we estimate each of the integrands in Equation (2.6). Assuming first that $m$ is odd and writing

$$
J_{x t}(X)=-B_{x,-t}^{*}\left(X B_{x,-t}+B_{x,-t} X\right)-\left(B_{x,-t}^{*} X+X B_{x,-t}^{*}\right) B_{x,-t}
$$

we use the canonical anti-commutation relations to contract exactly one pair of legs, in all possible ways, in each of the two anticommutators in brackets. This yields

$$
J_{x t}(X)=\sum_{i, j}\left\langle f_{x,-t}^{i}, g^{j}\right\rangle P_{i j}
$$

where each $P_{i j}$ is a Wick monomial of degree $(m+2 n-2)$ and the series has $2 m n$ terms. If $t_{0} \geqq 0$ Fourier analysis yields the estimate

$$
\left|\left\langle f_{x,-t}^{i}, g^{j}\right\rangle\right| \leqq C_{0}(1+|x|)^{-4}
$$


valid for all $0 \leqq t \leqq t_{0}$ and $x \in \mathbb{R}^{3}$. Equations (2.8) and (2.9) are adequate to control one pair of space and time integrations in Equation (2.6). Applying Equation (2.8) iteratively and finally estimating the uncontracted field operators in norm we obtain

$$
\begin{aligned}
\left\|\int \ldots \int J_{x_{1} s_{1}} \ldots J_{x_{n} s_{n}} X d x_{1} \ldots d x_{r} d s_{1} \ldots d s_{r}\right\| \\
\leqq a c^{r} \int_{s_{1}=0}^{t} \ldots \int_{s_{n}=0}^{s_{n}-1} 2 m n \cdot 2(m+2 n-2) n \cdot 2(m+4 n-4) n \ldots \\
\quad 2\{m+(2 r-2) n-(2 r-2)\} n d s_{1} \ldots \mathrm{ds} s_{r} \\
\leqq a 2^{r} c^{r} n^{r} t^{r} m(m+2 n-2) \ldots\{m+(2 r-2) n-(2 r-2)\} / r !
\end{aligned}
$$

valid for all $0 \leqq t \leqq t_{0}$, where $a$ depends on $X$ but $c$ does not. By the ratio test the series in Equation (2.6) is norm convergent if $0 \leqq t \leqq t_{0}$ and $4 c n^{2} t<1$. A similar conclusion for even values of $m$ can be based on the formula

$$
J_{x t}(X)=B_{x,-t}^{*}\left(X B_{x,-t}-B_{x,-t} X\right)+\left(B_{x,-t}^{*} X-X B_{x,-t}^{*}\right) B_{x,-t} .
$$

It follows that if $X$ is of the form of Equation (2.7) and

$$
0 \leqq t \leqq t_{1} \equiv \min \left\{t_{0},\left(8 c n^{2}\right)^{-1}\right\}
$$

then the right-hand side of Equation (2.5) converges uniformly to the right-hand side of Equation (2.6). The density of linear combinations of $\operatorname{such} X$, combined with the estimates

$$
\left\|S_{t}\right\|=1, \quad\left\|T_{a}(t)\right\| \leqq 1
$$

valid for all $t \geqq 0$, implies that

$$
\lim _{a \rightarrow \infty} \sup _{0 \leqq t \leqq t_{1}}\left\|T_{a}(t) X-T(t) X\right\|=0
$$

for all $X \in \mathscr{A}$. The semigroup property of each $T_{a}(t)$ allows one to define $T(t)$ for all $t \geqq 0$ and extend the validity of Equation (2.10) to arbitrary $t_{1} \geqq 0$. This then implies that $T(t)$ is a dynamical semigroup on $\mathscr{A}$.

By Equation (2.6) the formula

$$
T(t) \alpha_{x}(X)=\alpha_{x} T(t) X
$$

is valid for $0 \leqq t \leqq t_{1}$ and $X$ of the form of Equation (2.7). Its validity for all $X \in \mathscr{A}$ follows by density, and for all $t \geqq 0$ follows by the semigroup property of $T(t)$.

It is well-known with reversible evolution equations that even when it is possible to define the dynamics as an automorphism group of the appropriate $C^{*}$-algebra, it may not be possible to make sense of the formal Hamiltonian of the system unless one excludes spatially homogeneous pure creation terms from the interaction Hamiltonian $[28,49]$. The situation with irreversible evolution equations is similar, as we can see from an earlier paper [9], where in order to integrate an evolution equation similar to Equation (2.1) within the Fock representation of the CAR's, the further restriction that $B$ was of the form

$$
B=a^{\#}\left(f^{1}\right) \ldots a^{\#}\left(f^{n-1}\right) a\left(f^{n}\right)
$$


was necessary. An associated difference between our approach and that of [9] is that here we are defining the time evolution of observables rather than states.

\section{§3. Evolution of $G$-invariant States}

Let $\mathscr{A}$ be a separable $C^{*}$-algebra and $G$ a group of automorphisms of $\mathscr{A}$ which is $G$ abelian in the sense of $[19,42]$. For example $G$ might be the group of space translations of the CAR algebra $\mathscr{A}$ over $L^{2}\left(\mathbb{R}^{3}\right)$, which is weakly asymptotically abelian by [16].

By $[19$, p. $222 ; 42$, p. 158$]$ the set $\mathscr{S}_{G}$ of $G$-invariant states on $\mathscr{A}$ is a simplex. The set $X$ of extreme points of $\mathscr{S}_{G}$ is a Borel set in $\mathscr{S}_{G}$ and hence a standard Borel space. We denote by $B(X)$ the set of bounded measurable functions on $X$ and by $P(X)$ the set of probability measures on $X$.

If we regard $X$ as being a classical macroscopic phase space for $\mathscr{A}$, the following theorem constructs a Markov process on $X$ which describes macroscopic irreversible dynamics compatible with $T_{t}$.

Theorem 3.1. If $T_{t}$ is a $G$-invariant dynamical semigroup on $\mathscr{A}$, there is a function $P_{t}(x, E)$ defined for all $t \geqq 0, x \in X$ and Borel sets $E \subseteq X$ such that

(i) $x, t \rightarrow P_{t}(x, E)$ is measurable for all $E$;

(ii) $E \rightarrow P_{t}(x, E)$ is a probability measure on $X$ for all $x, t$;

(iii) $\int_{X} P_{s}(x, d y) P_{t}(y, E)=P_{s+t}(x, E)$

for all $s, t, x, E$;

(iv) $x\left(T_{t} A\right)=\int_{X} \phi(A) P_{t}(x, d \phi)$

for all $x, t$ and $A \in \mathscr{A}$.

Proof. The barycentre map $B: P(X) \rightarrow \mathscr{S}_{G}$ is defined by

$$
f(B \mu)=\int_{X} f(x) \mu(d x)
$$

where $f$ is an arbitrary element of the space $A\left(\mathscr{S}_{G}\right)$ of continuous affine functions on $\mathscr{S}_{G}$. Since $\mathscr{S}_{G}$ is a simplex, $B$ is one-one onto [2, p. 87]. Since $T$ is $G$-invariant $T_{t}^{*}\left(\mathscr{S}_{G}\right)$ $\subseteq \mathscr{T}_{G}$ and we can therefore define

$$
P_{t}(x, E)=\left(B^{-1} T_{t}^{*} B \varepsilon_{x}\right)(E)
$$

where $\varepsilon_{x} \in P(X)$ is the point evaluation at $x$. The validity of (ii), (iii) and (iv) is immediate.

If $\mathscr{D}$ is the norm dense subspace of $A\left(\mathscr{S}_{G}\right)$ obtained by restricting elements of $\mathscr{A}$ to $\mathscr{S}_{G}$, then by Equation (3.1)

$$
x, t \rightarrow \int_{X} f(\phi) P_{t}(x, d \phi)
$$

is measurable for all $f \in \mathscr{D}$, and hence for all $f \in A\left(\mathscr{S}_{G}\right)$. Identifying the monotone sequential envelope of $A\left(\mathscr{S}_{G}\right)$ with $B(X)$ by [1], it follows that Equation (3.2) defines a measurable function of $x, t$ for all $f \in B(X)$. Choosing $f$ to be the characteristic function of $E$ completes the proof of (i). 


\section{§4. Spatial Implementation of Dynamics}

If $T_{t}$ is a dynamical semigroup on $\mathscr{A}$ and $\pi$ is a representation of $\mathscr{A}$ on a Hilbert space $\mathscr{H}$, we say that $\pi$ is spatial with respect to $T_{t}$ if there is a one-parameter semigroup $T_{t}^{-}$of normal completely positive maps on the von Neumann algebra $\mathscr{V}=(\pi \mathscr{A})^{\prime \prime}$ such that

$$
T_{t}^{-}(\pi X)=\pi\left(T_{t} X\right)
$$

for all $X \in \mathscr{A}$. We say that $\pi$ is fully spatial with respect to $T_{t}$ if there is a oneparameter semigroup of normal completely positive maps $T_{t}^{\prime}$ on $\mathscr{L}(\mathscr{H})$ such that

$$
T_{t}^{\prime}(\pi X)=\pi\left(T_{t} X\right)
$$

for all $X \in \mathscr{A}$. Unlike $T_{t}^{\prime}$, the semigroup $T_{t}^{-}$is uniquely determined by $T_{t}$ when it exists. We say that a state $\phi$ on $\mathscr{A}$ is (fully) spatial with respect to $T_{t}$ if the cyclic representation associated with $\phi$ has this property. It is well-known that every invariant state is fully spatial with respect to a dynamical group, but the same does not hold for dynamical semigroups even when the algebra $\mathscr{A}$ is finite-dimensional.

Example 4.1. Let $\mathscr{A}=M(2, \mathbb{C}) \oplus M(2, \mathbb{C})$ and define $P \in \mathscr{A}$ and $\tau, \phi \in \mathscr{S}$ by

$$
\begin{aligned}
& P=\left(\begin{array}{ll}
0 & 0 \\
0 & 0
\end{array}\right) \oplus\left(\begin{array}{ll}
0 & 0 \\
0 & 1
\end{array}\right) \\
& \tau\left\{\left(\begin{array}{ll}
a & b \\
c & d
\end{array}\right) \oplus\left(\begin{array}{ll}
a^{\prime} & b^{\prime} \\
c^{\prime} & d^{\prime}
\end{array}\right)\right\}=\frac{1}{2}(a+d) \\
& \phi\left\{\left(\begin{array}{ll}
a & b \\
c & d
\end{array}\right) \oplus\left(\begin{array}{ll}
a^{\prime} & b^{\prime} \\
c^{\prime} & d^{\prime}
\end{array}\right)\right\}=a^{\prime} .
\end{aligned}
$$

The evolution equation

$$
\frac{d X}{d t}=\tau(X) P-\frac{1}{2}(P X+X P)
$$

on $\mathscr{A}$ has solution $X(t)=T_{t} X$ where $T_{t}$ is a dynamical semigroup on $\mathscr{A}$. Since

$$
\phi\left\{\tau(X) P-\frac{1}{2}(P X+X P)\right\}=0
$$

for all $X \in \mathscr{A}, \phi$ is an invariant state on $\mathscr{A}$. The GNS representation associated with $\phi$ is two-dimensional with kernel

$$
\mathscr{I}=\left\{\left(\begin{array}{ll}
a & b \\
c & d
\end{array}\right) \oplus\left(\begin{array}{ll}
0 & 0 \\
0 & 0
\end{array}\right): a, b, c, d \in \mathbb{C}\right\} .
$$

It is clear from the evolution equation that $\mathscr{I}$ is not invariant under $T_{t}$ so there is not any induced semigroup on $\mathscr{A} / \mathscr{I}$.

We recall that a state $\phi$ on $\mathscr{A}$ is called faithful if $\phi\left(X^{*} X\right)=0$ implies $X=0$. It is called separating if $\pi(\mathscr{A})^{\prime} \Omega$ is dense in $\mathscr{H}$, where $\pi$ is the cyclic representation on $\mathscr{H}$ and $\Omega$ the cyclic vector associated with $\phi$. If $\mathscr{A}$ is simple every separating state is faithful. If $\phi$ is a KMS state with respect to any automorphism group of $\mathscr{A}$ then $\phi$ is separating [51, p. 69]. 
Theorem 4.2. If $\phi$ is a separating invariant state on the $C^{*}$-algebra $\mathscr{A}$ with respect to the dynamical semigroup $T_{t}$, then $\phi$ is spatial with respect to $T_{t}$.

Proof. The normal folium $\mathscr{N}$ of $\phi$ is defined [25] as the set of states $\psi$ on $\mathscr{A}$ which can be put in the form

$$
\psi(X)=\operatorname{tr}[\varrho \pi(X)]
$$

for some operator $\varrho \geqq 0$ on $\mathscr{H}$ such that $\operatorname{tr}[\varrho]=1$. Alternatively $[15$, p. 38] $\mathscr{N}$ is the set of states on $\mathscr{A}$ which may be extended to normal states on $\mathscr{V}=(\pi \mathscr{A})^{\prime \prime}$. We claim that

$$
\mathscr{N}=\{\psi \in \mathscr{S}: \psi \leqq \alpha \phi \text { for some } \alpha\}^{-}
$$

the closure being in norm. Both sides of Equation (4.1) are norm closed convex sets. If $0 \leqq \psi \leqq \alpha \phi$ then by the GNS construction there is an operator $A \in \mathscr{V}^{\prime}$ such that $0 \leqq A \leqq \alpha 1$ and

$$
\psi(X)=\langle\pi(X) A \Omega, \Omega\rangle
$$

for all $X \in \mathscr{A}$. Thus

$$
\psi(X)=\left\langle\pi(X)\left(A^{1 / 2} \Omega\right),\left(A^{1 / 2} \Omega\right)\right\rangle
$$

so $\psi \in \mathscr{N}$ and the RHS of Equation (4.1) is contained in the LHS. Conversely since $\phi$ is separating given $v \in \mathscr{H}$ with $\|v\|=1$ and given $\varepsilon>0$ there exists $A \in \mathscr{V}$ with $\|A \Omega-v\|<\varepsilon$ and $\|A \Omega\|=1$. Defining the state $\psi$ on $\mathscr{A}$ by

$$
\psi(X)=\langle\pi(X)(A \Omega),(A \Omega)\rangle
$$

we see that

$$
0 \leqq \psi \leqq\left\|A^{*} A\right\| \phi
$$

Since $\varepsilon>0$ is arbitrary the RHS of Equation (4.1) contains all vector states and hence all states which can be represented by density matrices $\varrho$.

The invariance of $\phi$, the positivity of $T_{t}$, and Equation (4.1) imply that there are positive linear maps $R_{t}$ on the Banach subspace $\mathscr{B}=\operatorname{lin}(\mathscr{N})$ of $\mathscr{A}^{*}$ such that

$$
\left(R_{t} \psi\right)(X)=\psi\left(T_{t} X\right)
$$

for all $X \in \mathscr{A}, \psi \in \mathscr{B}$ and $t \geqq 0$.

Identifying $\mathscr{B}^{*}$ with $\mathscr{V}$ in the canonical manner $[15$, p. 38; 19, p.119] there are normal positive linear maps $T_{t}^{-}=R^{*}$ on $\mathscr{V}$ such that

$$
T_{t}^{-}(\pi X)=\pi\left(T_{t} X\right)
$$

for all $X \in \mathscr{A}$ and $t \geqq 0$. The complete positivity of $T_{t}$ implies that $T_{t}^{-}$is completely positive and the semigroup property of $T_{t}$ implies that $T_{t}^{-}$is a semigroup.

We conjecture that $\phi$ need not be fully spatial with respect to $T_{t}$ in the circumstances of the above theorem.

Theorem 4.3. Let $\phi$ be a separating type 1 factor state of the $C^{*}$-algebra $\mathscr{A}$, invariant with respect to the dynamical semigroup $T_{t}$. Then $\phi$ is fully spatial with respect to $T_{t}$. 
Proof. By [15, p. 124] the Hilbert space $\mathscr{H}$ of the cyclic representation associated to $\phi$ may be written in the form $\mathscr{H}=\mathscr{H}_{1} \otimes \mathscr{H}_{2}$ so that

$$
(\pi \mathscr{A})^{\prime \prime}=\mathscr{L}\left(\mathscr{H}_{1}\right) \otimes 1 .
$$

The maps $T_{t}^{-}$of Theorem 4.2 may therefore be considered as completely positive maps on $\mathscr{L}\left(\mathscr{H}_{1}\right)$ and by $[12$, p. $140 ; 32]$ have representations

$$
T_{t}^{-}(X)=\sum_{n=1}^{\infty} A_{n t} X A_{n t}^{*}
$$

where

$$
\sum_{n=1}^{\infty} A_{n t} A_{n t}^{*}=1
$$

the sums being convergent in the weak operator topology.

If we define $T_{t}^{\prime}: \mathscr{L}(\mathscr{H}) \rightarrow \mathscr{L}(\mathscr{H})$ by

$$
T_{t}^{\prime}(X)=\sum_{n=1}^{\infty}\left(A_{n t} \otimes 1_{\mathscr{H}_{2}}\right) X\left(A_{n t} \otimes 1_{\mathscr{H}_{2}}\right)^{*}
$$

then each $T_{t}^{\prime}$ is normal and completely positive. Subject to normality $T_{t}^{\prime}$ is uniquely determined by

$$
T_{t}^{\prime}(A \otimes B)=T_{t}^{-}(A) \otimes B
$$

valid for all $A \in \mathscr{L}\left(\mathscr{H}_{1}\right)$ and $B \in \mathscr{L}\left(\mathscr{H}_{2}\right)$. Equation (4.2) implies that $T_{t}^{\prime}$ is a oneparameter semigroup.

In the remainder of this section we apply some of the abstract results above to the dynamical semigroups constructed on the CAR algebra $\mathscr{A}$ in Section 2.

Theorem 4.4. Let $\phi$ be a spatially homogeneous state on the CAR algebra $\mathscr{A}$, which is invariant with respect to the free dynamics $S_{t}$. Let $T_{t}$ be the dynamical semigroup associated with the evolution equation

$$
\begin{aligned}
\frac{d X}{d t}= & D(X)+\int_{\mathbb{R}^{3}}\left\{2 B_{x}^{*} \theta(X) B_{x}-B_{x}^{*} B_{x} X\right. \\
& \left.-X B_{x}^{*} B_{x}+2 \alpha B_{x} \theta(X) B_{x}^{*}-\alpha B_{x} B_{x}^{*} X-\alpha X B_{x} B_{x}^{*}\right\} d^{3} x
\end{aligned}
$$

as in Theorem 2.1, where $\alpha \geqq 0$. If

$$
\phi(X B)=\alpha \phi(B X)
$$

for all $X \in \mathscr{A}$ then $\phi$ is invariant with respect to $T_{t}$.

Proof. If we define

$$
\begin{aligned}
J_{x}(X)= & 2 B_{x}^{*} \theta(X) B_{x}-B_{x}^{*} B_{x} X-X B_{x}^{*} B_{x} \\
& +2 \alpha B_{x} \theta(X) B_{x}^{*}-\alpha B_{x} B_{x}^{*} X-\alpha X B_{x} B_{x}^{*}
\end{aligned}
$$

then

$$
\phi\left(J_{x} X\right)=0
$$


for all $x \in \mathbb{R}^{3}$ and $X \in \mathscr{A}_{0}$ by the spatial homogeneity of $\phi$ and Equation (4.3). If $X \in \mathscr{A}_{1}$ then $J_{x} X \in \mathscr{A}_{1}$ so Equation (4.4) still holds by [42, p.178]. Therefore Equation (4.4) is valid for all $x \in \mathbb{R}^{3}$ and $X \in \mathscr{A}$. By Equation (2.4)

$$
\phi\left(T_{a}(t) X\right)=\phi(X)
$$

for all $X \in \mathscr{A}, t \geqq 0$ and $a \geqq 0$. Letting $\alpha \rightarrow \infty$ we obtain

$$
\phi(T(t) X)=\phi(X)
$$

for all $X \in \mathscr{A}$ and $t \geqq 0$.

For the application of this theorem we recall that the universally invariant state $\phi_{\beta}$ at the inverse temperature $\beta$ is defined formally by

$$
\phi_{\beta}(X)=\operatorname{tr}\left[e^{-\beta N} X\right] / \operatorname{tr}\left[e^{-\beta N}\right]
$$

where $N$ is the number operator [45].

Theorem 4.5. If the Wick monomial $B$ is of odd degree and involves $m$ creation and $n$ annihilation terms, then $\phi_{\beta}$ is invariant and spatial with respect to $T_{t}$ if

$$
e^{\beta(m-n)}=\alpha .
$$

Proof. We note that $\phi_{\beta}$ is a KMS state with respect to the automorphism group $\alpha\left(e^{i t} 1\right)$ of $\mathscr{A}$. The verification of Equation (4.3) is a consequence of the KMS property; formally

$$
\begin{aligned}
\phi_{\beta}(X B) & =\operatorname{tr}\left[e^{-\beta N} X N\right] / \operatorname{tr}\left[e^{-\beta N}\right] \\
& =\operatorname{tr}\left[e^{-\beta N}\left(e^{\beta N} B e^{-\beta N}\right) X\right] / \operatorname{tr}\left[e^{-\beta N}\right] \\
& =e^{\beta(m-n)} \operatorname{tr}\left[e^{-\beta N} B X\right] / \operatorname{tr}\left[e^{-\beta N}\right] \\
& =\alpha \phi_{\beta}(B X) .
\end{aligned}
$$

The KMS property also implies that $\phi_{\beta}$ is a separating state $\left[51\right.$, p. 69], so $\phi_{\beta}$ is spatial by Theorem 4.2.

Comment. It should be possible to extend Theorem 4.5 to the case where $\phi$ is a more general KMS state and the evolution equation has a suitable form, dependent on $\phi$.

\section{§5. An Exactly Soluble Evolution Equation}

Many of the above ideas can be well illustrated with the exactly soluble evolution equation

$$
\begin{aligned}
\frac{d X}{d t}= & D_{S}(X) \\
& +\int_{\mathbb{R}^{3}}\left\{2 a^{*}\left(f_{x}\right) \theta(X) a\left(f_{x}\right)-a^{*}\left(f_{x}\right) a\left(f_{x}\right) X-X a^{*}\left(f_{x}\right) a\left(f_{x}\right)\right\} d^{3} x \\
& +\int_{\mathbb{R}^{3}}\left\{2 a\left(g_{x}\right) \theta(X) a^{*}\left(g_{x}\right)-a\left(g_{x}\right) a^{*}\left(g_{x}\right) X-X a\left(g_{x}\right) a^{*}\left(g_{x}\right)\right\} d^{3} x
\end{aligned}
$$

on the CAR algebra $\mathscr{A}$ over $\mathscr{H}=L^{2}\left(\mathbb{R}^{3}\right)$. The three terms on the RHS of Equation (5.1) are interpreted respectively as the free generator, a stochastic term tending to annihilate particles, and a stochastic term tending to create particles. 
It is advantageous to consider this model at the maximum level of generality. We let $\mathscr{A}^{\prime}$ be the linear span of all Wick monomials, this being a dense *-subalgebra of $\mathscr{A}$. We let $\mathscr{A}^{\prime \prime}$ be the linear span of all Wick monomials whose test functions lie in the domain of an unbounded self-adjoint operator $S$ on $\mathscr{H}$. The derivation $D_{S}$ on $\mathscr{A}$ was defined in Section 1 and has domain containing $\mathscr{A}^{\prime \prime}$. We turn now to the definition of abstract versions of the two stochastic terms of Equation (5.1)

Theorem 5.1. For every bounded operator $A$ on $\mathscr{H}$ such that $A+A^{*} \geqq 0$ there is a linear operator $Z_{A}: \mathscr{A}^{\prime} \rightarrow \mathscr{A}$ which depends linearly on $A$ and has the further properties.

(i) If $A$ is trace class $Z_{A}$ may be extended to a bounded linear operator on $\mathscr{A}$ which is the generator of a norm continuous, completely positive, one-parameter semigroup on $\mathscr{A}$.

(ii) If $A$ is bounded the closure of $Z_{A}$ is the generator of a strongly continuous, completely positive one-parameter semigroup $T(t)$ on $\mathscr{A}$ such that

$$
T(t)\left\{a^{\#}\left(f^{1}\right) \ldots a^{\#}\left(f^{n}\right)\right\}=a^{\#}\left(e^{-A t} f^{1}\right) \ldots a^{\#}\left(e^{-A t} f^{n}\right)
$$

for all normal ordered Wick monomials.

Proof. If $A$ is trace class then applying the spectral theorem to its skew- and selfadjoint parts we obtain

$$
A=\sum_{n=1}^{\infty} \lambda_{n}\left|g^{n}\right\rangle\left\langle g^{n}\left|+i \sum_{n=1}^{\infty} \mu_{n}\right| h^{n}\right\rangle\left\langle h^{n}\right|
$$

where $\left\|g^{n}\right\|=\left\|h^{n}\right\|=1$ for all $n, \mu_{n}$ are real, $\lambda_{n} \geqq 0$ and

$$
\sum_{n=1}^{\infty} \lambda_{n}<\infty, \quad \sum_{n=1}^{\infty}\left|\mu_{n}\right|<\infty .
$$

The formula

$$
\begin{aligned}
& Z_{A}(X)=\sum_{n=1}^{\infty} \lambda_{n}\left\{-a^{*}\left(g^{n}\right) a\left(g^{n}\right) X+2 a^{*}\left(g^{n}\right) \theta(X) a\left(g^{n}\right)-X a^{*}\left(g^{n}\right) a\left(g^{n}\right)\right\} \\
& -i \sum_{n=1}^{\infty} \mu_{n}\left\{a^{*}\left(h^{n}\right) a\left(h^{n}\right) X-X a^{*}\left(h^{n}\right) a\left(h^{n}\right)\right\}
\end{aligned}
$$

is norm convergent and defines a linear operator $Z_{A}$ on $\mathscr{A}$ with

$$
\left\|Z_{A}\right\| \leqq \sum_{n=1}^{\infty}\left\{4 \lambda_{n}+2\left|\mu_{n}\right|\right\}<\infty .
$$

The complete positivity of the norm continuous semigroup $T_{t}=e^{Z_{A} t^{t}}$ follows from $[12$, p. $143 ; 33]$.

Direct calculations show that for any normal ordered Wick monomial

$$
\begin{aligned}
& Z_{\boldsymbol{A}}\left\{a^{\#}\left(f^{1}\right) \ldots a^{\#}\left(f^{n}\right)\right\} \\
& =-\sum_{j=1}^{n}\left[\left\{\prod_{i<j} a^{\#}\left(f^{i}\right)\right\} a^{\#}\left(A f^{j}\right)\left\{\prod_{i>j} a^{\#}\left(f^{i}\right)\right\}\right] .
\end{aligned}
$$


Together with the density of $\mathscr{A}^{\prime}$ in $\mathscr{A}$, this shows that $Z_{A}$ depends only on $A$, and not on the decomposition of $A$ in Equation (5.3). Equation (5.5) also implies that Equation (5.2) holds for any normal ordered Wick monomial.

We prove the theorem for arbitrary bounded $A$ such that $A+A^{*} \geqq 0$ by taking limits. If $A_{n}$ is a sequence of trace class operators such that $A_{n}+A_{n}^{*} \geqq 0$ and $A_{n} \rightarrow A$ strongly as $n \rightarrow \infty$, and if we define

$$
T_{n}(t)=\exp \left\{Z_{A_{n}} t\right\}
$$

then

$$
\lim _{n \rightarrow \infty} T_{n}(t)\left\{a^{\#}\left(f^{1}\right) \ldots a^{\#}\left(f^{n}\right)\right\}=a^{\#}\left(e^{-A t} f^{1}\right) \ldots a^{\#}\left(e^{-A t} f^{n}\right)
$$

for every normal ordered Wick monomial, the norm convergence being uniform for $t$ in any bounded interval. Because $\left\|T_{n}(t)\right\| \leqq 1$ for all $n$ and all $t \geqq 0$, there is a bounded operator $T(t): \mathscr{A} \rightarrow \mathscr{A}$ such that

$$
\lim _{n \rightarrow \infty} \sup _{0 \leqq t \leqq t_{0}}\left\|T_{n}(t) X-T(t) X\right\|=0
$$

for all $X \in \mathscr{A}$ and $t_{0} \geqq 0$. It is an immediate consequence of Equation (5.6) that $T(t)$ is a strongly continuous, completely positive, one parameter semigroup on $\mathscr{A}$ and that Equation (5.2) holds.

By Equation (5.2) the infinitesimal generator $Z_{A}$ of $T(t)$ has domain containing all normal ordered Wick monomials, on which it is given by Equation (5.5). Thus $\mathscr{A}^{\prime}$ is contained in the domain of $Z_{A}$, is invariant under $T(t)$ and so is a core for $Z_{A}$ by $[39$, p. 241].

Comments (i). An alternative proof of parts of the above theorem could be based on the fact proved in $[21,29,44]$ that since $\left\|e^{-A t}\right\| \leqq 1$ there exists a completely positive map $T_{A}(t)$ on $\mathscr{A}$ satisfying Equation (5.2) for all normal ordered Wick monomials.

(ii) It is an immediate consequence of the theorem that $T(t)$ leaves the observable algebra $\mathscr{A}_{0}$ invariant.

(iii) The theorem can be modified by supposing Equation (5.2) is satisfied for all anti-normal ordered Wick momials, and replacing $Z_{A}$ by an operator $Y_{A}$. In the proof Equation (5.4) is replaced by

$$
\begin{aligned}
& Y_{A}(X)=\sum_{n=1}^{\infty} \lambda_{n}\left\{-a\left(g^{n}\right) a^{*}\left(g^{n}\right) X+2 a\left(g^{n}\right) \theta(X) a^{*}\left(g^{n}\right)\right. \\
& \left.-X a\left(g^{n}\right) a^{*}\left(g^{n}\right)\right\}+i \sum_{n=1}^{\infty} \mu_{n}\left\{a\left(h^{n}\right) a^{*}\left(h^{n}\right) X-X a\left(h^{n}\right) a^{*}\left(h^{n}\right)\right\}
\end{aligned}
$$

while Equation (5.5) is replaced by

$$
\begin{aligned}
& Y_{A}\left\{a^{\#}\left(f^{1}\right) \ldots a^{\#}\left(f^{n}\right)\right\} \\
& =-\sum_{j=1}^{n}\left[\left\{\prod_{i<j} a^{\#}\left(f^{i}\right)\right\} a^{\#}\left(A f^{j}\right)\left\{\prod_{i>j} a^{\#}\left(f^{i}\right)\right\}\right]
\end{aligned}
$$


valid for all anti-normal ordered Wick monomials. The action of $Y_{A}$ on normal ordered Wick monomials may be deduced from Equation (5.7), but the expression is not so simple.

We combine the above results into one theorem.

Theorem 5.2. Let $S$ be an unbounded self-adjoint operator on $\mathscr{H}$ and $A, B$ bounded operators such that

$$
A+A^{*} \geqq 0, \quad B+B^{*} \geqq 0 .
$$

Then there exists a dynamical semigroup $T(t)$ on $\mathscr{A}$ whose infinitesimal generator $W$ is given on $\mathscr{A}^{\prime \prime}$ by

$$
W(X)=D_{S}(X)+Z_{A}(X)+Y_{B}(X) .
$$

\section{Moreover}

$$
T(t) X=\lim _{r \rightarrow \infty}\left(e^{D_{S} t r} e^{Z_{A} t / r} e^{Y_{B} t / r}\right)^{r} X
$$

for all $X \in \mathscr{A}^{\prime \prime}$ and $t \geqq 0$.

Proof. Our method will be directly related to the computation of $T(t)$. We define $\otimes_{m, n} \mathscr{H}$ as the projective tensor product [43, p. 36] of $m$ copies of $\mathscr{H}$ and $n$ copies of the complex conjugate Hilbert space $\mathscr{H}^{-}$. This is the Banach space completion of the algebraic tensor product for the norm

$$
\|\mid x\| \|=\inf \left\{\sum_{r=1}^{k}\left\|f_{1}^{r}\right\| \ldots\left\|f_{m+n}^{r}\right\|: x=\sum_{r=1}^{k} f_{1}^{r} \otimes \ldots \otimes f_{m+n}^{r}\right\} .
$$

We then define $\mathscr{A}_{N}^{\tilde{N}}=\sum_{m+n \leqq N} \otimes_{m, n} \mathscr{H}$.

There are unique linear contractions $i_{m, n}$ from $\otimes_{m, n} \mathscr{H}$ into $\mathscr{A}$ such that

$$
i_{m, n}\left(f_{1} \otimes \ldots \otimes f_{m+n}\right)=a^{*}\left(f_{1}\right) \ldots a^{*}\left(f_{m}\right) a\left(f_{m+1}\right) \ldots a\left(f_{m+n}\right)
$$

and these may be combined into one linear contraction $i_{N}: \mathscr{A}_{N} \rightarrow \mathscr{A}$. Our plan will be to construct the semigroup first in the $\mathscr{A}_{N}$ and then to carry it through to $\mathscr{A}$ using the Trotter product formula.

There is a strongly continuous one-parameter group of isometries $S_{t}^{\sim}$ on $\mathscr{A}_{N}^{\tilde{N}}$ such that for all $m, n$ we have

$$
S_{t}^{\sim}\left(f_{1} \otimes \ldots \otimes f_{m+n}\right)=\left(e^{i S t} f_{1}\right) \otimes \ldots \otimes\left(e^{i S t} f_{m+n}\right) .
$$

It is clear from Equations (1.1) and (1.5) that

$$
i_{N} S_{t}^{\sim} x=S_{t} i_{N} x
$$

for all $x \in \mathscr{A}_{N}$ and all $t \geqq 0$. The linear span $\mathscr{D}_{N}^{\tilde{N}}$ of all elementary tensor products $f_{1} \otimes \ldots \otimes f_{m+n}$ such that each $f_{i}$ lies in $\operatorname{dom}(S)$ is a core for the infinitesimal generator $\mathscr{D}_{\tilde{S}}^{\tilde{S}}$ of $S_{t}^{\sim}$ by [39, p. 241]. There is a unique bounded linear map $Z_{\boldsymbol{A}}^{\tilde{A}}$ on $\mathscr{A}_{N} \tilde{\text { such that }}$

$$
Z_{\dot{A}}^{\sim}\left(f_{1} \otimes \ldots \otimes f_{m+n}\right)=-\sum_{j=1}^{m+n}\left[\left\{\bigotimes_{i<j} f_{i}\right\} \otimes\left(A f_{j}\right) \otimes\left\{\bigotimes_{i>j} f_{i}\right\}\right] .
$$


One sees by Equation (5.5) that

$$
i_{N} Z_{A}^{\sim} x=Z_{A} i_{N} x
$$

for all $x \in \mathscr{A}_{N}^{\tilde{N}}$. Using Equation (5.7) to write down the action of $Y_{B}$ on normal ordered Wick monomials one may show similarly that there is a bounded operator $Y_{B}^{\sim}$ on $\mathscr{A}_{N}^{\sim}$

such that

$$
i_{N} Y_{B}^{\sim} x=Y_{B} i_{N} x
$$

for all $x \in \mathscr{A}_{N}^{\sim}$. Since $Z_{\boldsymbol{A}}^{\sim}$ and $Y_{B}^{\sim}$ are bounded there is a strongly continuous oneparameter semigroup $T_{N}^{\sim}(t)$ on $\mathscr{A}_{N}^{\sim}$ whose infinitesimal generator is equal to $\left(D_{S}^{\sim}+Z_{A}^{\sim}+Y_{B}^{\sim}\right)$ on its core $\mathscr{D}_{N}^{\sim}$. Moreover

$$
T_{N}^{\sim}(t) x=\lim _{r \rightarrow \infty}\left(e^{D \tilde{\boldsymbol{S}} t / r} e^{Z_{\tilde{A} t / r}} e^{Y_{\tilde{B}} t / r}\right)^{r} x
$$

for all $t \geqq 0$ and $x \in \mathscr{A}_{N}^{\sim}$ by [7]. It follows by Equations (5.10), (5.11) and (5.12) that the limit in Equation (5.9) exists for all $X$ in the dense subspace

$$
\bigcup_{N=1}^{\infty} i_{N} \mathscr{A}_{N}^{\sim}
$$

of $\mathscr{A}$. Since each of $e^{D_{S} t}, e^{Z_{A} t}, e^{Y_{B} t}$ is a completely positive contraction for all $t \geqq 0$, Equation (5.9) is proved for all $X \in \mathscr{A}$, and $T(t)$ is a completely positive contraction on $\mathscr{A}$ for all $t \geqq 0$. Moreover

$$
T(t) i_{N} x=i_{N} T_{N}^{\sim}(t) x
$$

for all $t \geqq 0$ and $x \in \mathscr{A}_{N}^{\sim}$. This formula implies that $T(t)$ is a strongly continuous oneparameter semigroup on $\mathscr{A}$. Applying $i_{N}$ to both sides of

$$
\lim _{t \rightarrow 0} t^{-1}\left(T_{N}^{\sim}(t) x-x\right)=\left(D_{\tilde{S}}^{\sim}+Z_{\boldsymbol{A}}^{\tilde{H}}+Y_{B}^{\sim}\right) x
$$

valid for all $x \in \mathscr{D}_{N}^{\sim}$, we obtain

$$
\lim _{t \rightarrow 0} t^{-1}(T(t) X-X)=\left(D_{S}+Z_{A}+Y_{B}\right) X
$$

for all $X \in \mathscr{A}^{\prime \prime}=\bigcup_{N=1}^{\infty} i_{N} \mathscr{D}_{N}^{\sim}$.

Comment. With some further work one could show that $\mathscr{A}^{\prime \prime}$ is a core for the infinitesimal generator $W$ of $T(t)$.

Corollary 5.3. If $f, g \in \mathscr{H}$ and

$$
C=i S-A-B
$$

then

$$
\begin{aligned}
& T(t)\left\{a^{*}(f) a(g)\right\}=a^{*}\left(e^{C t} f\right) a\left(e^{C t} g\right) \\
& +\int_{s=0}^{t}\left\langle\left(B+B^{*}\right) e^{C s} f, e^{C s} g\right\rangle d s \cdot 1
\end{aligned}
$$


Proof. We first note that $C$ is abounded perturbation of $i S$ and so is the generator of a one-parameter semigroup. Moreover $C$ is dissipative by Equation (5.8), so $e^{C t}$ is a contraction semigroup by $[12$, p. $103 ; 52$, p. 250$]$.

Since $T(t)$ are contractions it is sufficient to consider the case where $f$, $g \in \operatorname{dom}(S)$. Since

$$
\begin{aligned}
Y_{B}\left\{a^{*}(f) a(g)\right\} & =Y_{B}\left\{\langle f, g\rangle 1-a(g) a^{*}(f)\right\} \\
& =a(B g) a^{*}(f)+a(g) a^{*}(B f) \\
& =\langle f, B g\rangle \cdot 1-a^{*}(f) a(B g)+\langle B f, g\rangle \cdot 1-a^{*}(B f) a(g)
\end{aligned}
$$

we find that

$$
\begin{aligned}
& W\left\{a^{*}(f) a(g)\right\}=a^{*}(i S f) a(g)+a^{*}(f) a(i S g)-a^{*}(A f) a(g) \\
& -a^{*}(f) a(A g)+\langle f, B g\rangle \cdot 1+\langle f, B g\rangle \cdot 1-a^{*}(B f) a(g)-a^{*}(f) a(B g) \\
& =a^{*}(C f) a(g)+a^{*}(f) a(C g)+\left\langle\left(B+B^{*}\right) f, g\right\rangle \cdot 1 .
\end{aligned}
$$

This may be directly integrated yielding Equation (5.14).

Comment. One may similarly find explicit expressions for $T(t)$ applied to any Wick monomial. We shall, however, manage to avoid doing the necessary computations.

\section{§6. Quasi-free States}

We study the time evolution of quasi-free states of the CAR algebra under the dynamical semigroup $T(t)$ constructed in the last section. If $R$ is a bounded operator on $\mathscr{H}$ with $0 \leqq R \leqq 1$ then by $[5,14,34,45]$ there exists a unique state $\omega_{R}$ on $\mathscr{A}$ such that

$$
\omega_{R}\left\{a^{*}\left(f_{m}\right) \ldots a^{*}\left(f_{1}\right) a\left(g_{1}\right) \ldots a\left(g_{n}\right)\right\}=\delta_{m n} \operatorname{det}\left\{\left\langle R f_{i}, g_{j}\right\rangle\right\}
$$

for all normal ordered Wick monomials. These states are factor states and their quasi- and unitary equivalence as $R$ varies has been determined in [37]. The Fock state, anti-Fock state and central trace are given by $R=0, R=1$ and $R=\frac{1}{2}$ respectively.

Lemma 6.1. The map $R \rightarrow \omega_{R}$ is a homeomorphism of $\{A \in \mathscr{L}(\mathscr{H}): 0 \leqq A \leqq 1\}$ with the weak operator topology onto the closed set $\mathscr{S}_{Q F}$ of quasi-free states on $\mathscr{A}$ under the weak* topology

Proof. This is a trivial consequence of Equation (6.1).

Theorem 6.2. The semigroup $T_{t}^{*}$ maps $\mathscr{S}_{Q F}$ into itself. Indeed

$$
T_{t}^{*}\left(\omega_{R}\right)=\omega_{R(t)}
$$

where

$$
R(t)=e^{C * t} R e^{C t}+\int_{s=0}^{t} e^{C * s}\left(B+B^{*}\right) e^{C s} d s .
$$


Proof. By Equation (5.9) and the closedness of $\mathscr{S}_{Q F}$ it is sufficient for the first statement of the theorem to prove that $\mathscr{S}_{Q F}$ is invariant under each of $e^{D_{S} t}, e^{Z_{A} t}, e^{Y_{B} t}$.

$$
\begin{aligned}
& \left\{\left(e^{D s t}\right)^{*} \omega_{R}\right\}\left\{a^{*}\left(f_{m}\right) \ldots a^{*}\left(f_{1}\right) a\left(g_{1}\right) \ldots a\left(g_{n}\right)\right\} \\
& =\omega_{R}\left\{a^{*}\left(e^{i S t} f_{m}\right) \ldots a^{*}\left(e^{i S t} f_{1}\right) a\left(e^{i S t} g_{1}\right) \ldots a\left(e^{i S t} g_{n}\right)\right\} \\
& =\delta_{m n} \operatorname{det}\left\{\left\langle\operatorname{Re} e^{i S t} f_{i}, e^{i S t} g_{j}\right\rangle\right\} \\
& =\omega_{e^{-i S t}} \operatorname{Re}^{i S t}\left\{a^{*}\left(f_{m}\right) \ldots a\left(g_{n}\right)\right\}
\end{aligned}
$$

so

$$
\left(e^{D_{S} t}\right) * \omega_{R}=\omega_{e^{-\imath} S t} R^{i S t} .
$$

The proof that

$$
\left(e^{Z_{A t}}\right)^{*} \omega_{R}=\omega_{e^{-A^{*} t}} e^{-A t}
$$

is similar. To deal with $e^{Y_{B} t}$ we need the formula

$$
\begin{aligned}
& \omega_{R}\left\{a\left(g_{1}\right) \ldots a\left(g_{n}\right) a^{*}\left(f_{m}\right) \ldots a^{*}\left(f_{1}\right)\right\} \\
& =\delta_{m n} \operatorname{det}\left\{\left\langle(1-R) f_{i}, g_{j}\right\rangle\right\}
\end{aligned}
$$

for all anti-normal ordered Wick monomials, which may be deduced from Equation (6.1) by straightforward algebraic manipulations. By Equation (5.7)

$$
\begin{aligned}
& \left\{\left(e^{Y_{B} t}\right)^{*} \omega_{R}\right\}\left\{a\left(g_{1}\right) \ldots a\left(g_{n}\right) a^{*}\left(f_{m}\right) \ldots a^{*}\left(f_{1}\right)\right\} \\
& =\omega_{R}\left\{a\left(e^{-B t} g_{1}\right) \ldots a^{*}\left(e^{-B t} f_{1}\right)\right\} \\
& =\delta_{m n} \operatorname{det}\left\{\left\langle(1-R) e^{-B t} f_{i}, e^{-B t} g_{j}\right\rangle\right\} \\
& =\delta_{m n} \operatorname{det}\left\{\left\langle\left(1-\left[1-e^{-B^{* t}}(1-R) e^{-B t}\right]\right) f_{i}, g_{j}\right\rangle\right\} \\
& =\omega_{1-e^{-B * t}(1-R) e^{-B t}}\left\{a\left(g_{1}\right) \ldots a^{*}\left(f_{1}\right)\right\}
\end{aligned}
$$

so

$$
\left(e^{Y_{B} t}\right) * \omega_{R}=\omega_{1-e^{-B * t}(1-R) e^{-B t}} .
$$

Having established the validity of an equality of the form of Equation (6.2) the precise value of $R(t)$ may be deduced using Corollary 5.3. We have

$$
\begin{aligned}
& \langle R(t) f, g\rangle \\
& =\omega_{R(t)}\left\{a^{*}(f) a(g)\right\}=\left(T_{t}^{*} \omega_{R}\right)\left\{a^{*}(f) a(g)\right\} \\
& =\omega_{R}\left\{a^{*}\left(e^{C t} f\right) a\left(e^{C t} g\right)+\int_{s=0}^{t}\left\langle\left(B+B^{*}\right) e^{C s} f, e^{C s} g\right\rangle \cdot 1\right\} \\
& =\left\langle R e^{C t} f, e^{C t} g\right\rangle+\int_{s=0}^{t}\left\langle\left(B+B^{*}\right) e^{C s} f, e^{C s} g\right\rangle d s
\end{aligned}
$$

for all $f, g \in \mathscr{H}$. This yields Equation (6.3).

\section{§7. Equilibrium States}

Let $T_{t}$ be a dynamical semigroup on a $C^{*}$-algebra $\mathscr{A}$ and let $\phi$ be a state on $\mathscr{A}$ which is invariant with respect to $T_{t}^{*}$. There are various criteria for stability of $\phi$ which we now enumerate. 
(i) $\phi$ is globally stable if $T_{t}^{*} \psi \rightarrow \phi$ in the weak* topology as $t \rightarrow \infty$ for all $\psi \in \mathscr{S}$.

(ii) $\phi$ is weakly locally stable if it is spatial and $T_{t}^{*} \psi \rightarrow \phi$ in the weak* topology as $t \rightarrow \infty$ for all $\psi$ in the normal folium of $\phi$.

(iii) $\phi$ is strongly locally stable if it is spatial and $T_{t}^{*} \psi \rightarrow \phi$ is norm as $t \rightarrow \infty$ for all $\psi$ in the normal folium of $\phi$. Our condition of weak locally stability is close to that used in [41] and other local stability conditions may be found in $[3,26]$; all these refer to reversible dynamics.

Strong local stability is too strong a property to be relevant for dynamical groups. For if $T_{t}^{*} \psi \rightarrow \phi$ in norm as $t \rightarrow \infty$ then since

$$
\left\|T_{t}^{*} \psi-\phi\right\|=\left\|\psi-T_{-t}^{*} \phi\right\|=\|\psi-\phi\|
$$

it follows that $\psi=\phi$. We include for completeness a well-known criterion for weak local stability.

Theorem 7.1. If $T_{t}$ is a dynamical group on $\mathscr{A}$ and $\phi$ an invariant state which is clustering in the sense that

$$
\lim _{t \rightarrow \infty} \phi\left\{Y^{*}\left(T_{t} X\right) Z\right\}=\phi\left\{Y^{*} Z\right\} \phi\{X\}
$$

for all $X, Y, Z \in \mathscr{A}$, then $\phi$ is weakly locally stable.

Proof. If $\pi$ is the cyclic representation and $\Omega$ the cyclic vector associated with $\phi$ then

$$
\lim _{t \rightarrow \infty}\left\langle\pi\left(T_{t} X\right)(\pi Z \Omega),(\pi Y \Omega)\right\rangle=\langle\phi(X) 1(\pi Z \Omega),(\pi Y \Omega)\rangle
$$

for all $Y, Z \in \mathscr{A}$ so

$$
\lim _{t \rightarrow \infty} \pi\left(T_{t} X\right)=\phi(X) 1
$$

in the weak operator topology for all $X \in \mathscr{A}$. If $\psi$ lies in the normal folium of $\phi$ and $\varrho$ is a density matrix such that

$$
\psi(X)=\operatorname{tr}[\varrho \pi(X)]
$$

for all $X \in \mathscr{A}$, then

$$
\lim _{t \rightarrow \infty}\left(T_{t}^{*} \psi\right)(X)=\lim _{t \rightarrow \infty} \operatorname{tr}\left[\varrho \pi\left(T_{t} X\right)\right]=\operatorname{tr}[\varrho \phi(X) 1]=\phi(X)
$$

for all $X \in \mathscr{A}$.

We can provide examples of the other types of stability with the dynamical semigroups introduced in Theorem 5.2. Some preparatory lemmas will be needed.

Lemma 7.2. If $e^{C t}$ converges strongly to zero as $t \rightarrow \infty$ then there is exactly one temporally invariant quasi-free state, namely $\omega_{R(\infty)}$ where

$$
R(\infty)=\int_{0}^{\infty} e^{C * s}\left(B+B^{*}\right) e^{C s} d s
$$

satisfies $0 \leqq R(\infty) \leqq 1$. 
Proof. Equation (5.8) implies that $R(\infty) \geqq 0$ and also

$$
R(\infty) \leqq-\int_{0}^{\infty} e^{C * s}\left(C+C^{*}\right) e^{C s} d s=-\left[e^{C * t} e^{C t}\right]_{0}^{\infty}=1
$$

This calculation also establishes that the integral in Equation (7.2) is convergent in the weak operator topology. If $0 \leqq R \leqq 1$ and $R(t)$ is defined by Equation (6.3) then $R(t)$ converges in the weak operator topology to $R(\infty)$ as $t \rightarrow \infty$. This implies all the statements of the lemma by Lemma 6.1.

Lemma 7.3. Let $T(t)$ be a strongly continuous one parameter contraction semigroup on a Banach space $\mathscr{A}_{n}$ and let $\left\{\mathscr{A}_{r}\right\}_{r=0}^{n}$ be an increasing sequence of closed invariant subspaces of $\mathscr{A}_{n}$. If the induced semigroups on $\mathscr{A}_{r} / \mathscr{A}_{r-1}$ all converge strongly to zero as $t \rightarrow \infty$, then for all $X \in \mathscr{A}_{n}$ and $\varepsilon>0$ there exists $t_{0}$ such that if $t \geqq t_{0}$ then

$$
\|T(t) X-Y\|<\varepsilon
$$

for some $Y \in \mathscr{A}_{0}$ depending on $X, t$ and $\varepsilon$.

Proof. The truth of the lemma for $n=0$ is evident and we suppose inductively that it is true for $(n-1)$. Given $X \in \mathscr{A}_{n}$ and $\varepsilon>0$ let $t \geqq t_{0}$ imply that

$$
\left\|T(t)\left\{X+\mathscr{A}_{n-1}\right\}\right\| \mid<\varepsilon / 2
$$

where $\||\cdot|||$ is the quotient norm in $\mathscr{A}_{n} / \mathscr{A}_{n-1}$.

Then

$$
\left\|T\left(t_{0}\right) X-X^{\prime}\right\|<\varepsilon / 2
$$

for some $X^{\prime} \in \mathscr{A}_{n-1}$. Let $t_{1}$ be such that $t \geqq t_{1}$ implies

$$
\left\|T(t) X^{\prime}-X^{\prime \prime}\right\|<\varepsilon / 2
$$

for some $X^{\prime \prime} \in \mathscr{A}_{0}$. Then $t \geqq t_{0}+t_{1}$ implies

$$
\begin{aligned}
& \left\|T(t) X-X^{\prime \prime}\right\| \leqq\left\|T\left(t-t_{0}\right)\right\|\left\|T\left(t_{0}\right) X-X^{\prime}\right\| \\
& +\left\|T\left(t-t_{0}\right) X^{\prime}-X^{\prime \prime}\right\|<\varepsilon .
\end{aligned}
$$

This establishes the inductive step, and hence the lemma.

Theorem 7.4. If $e^{C t}$ converges strongly to zero as $t \rightarrow \infty$, then the state $\omega_{R(\infty)}$ is globally stable.

Proof. We let $\mathscr{A}_{n}$ be the norm closure in $\mathscr{A}$ of the set of Wick polynomials of degree $\leqq n$. For any Wick monomial

$$
W=a^{\#}\left(f^{1}\right) \ldots a^{\#}\left(f^{n}\right)
$$

the element $W+\mathscr{A}_{n-1}$ of $\mathscr{A}_{n} / \mathscr{A}_{n-1}$ is independent of the order of the creationannihilation operators in $W$. Therefore for such $W$

$$
\begin{aligned}
& e^{D_{S} t}\left(W+\mathscr{A}_{n-1}\right)=a^{\#}\left(e^{i S t} f^{1}\right) \ldots a^{\#}\left(e^{i S t} f^{n}\right)+\mathscr{A}_{n-1} \\
& e^{Z_{A} t}\left(W+\mathscr{A}_{n-1}\right)=a^{\#}\left(e^{-A t} f^{1}\right) \ldots a^{\#}\left(e^{-A t} f^{n}\right)+\mathscr{A}_{n-1} \\
& e^{Y_{B} t}\left(W+\mathscr{A}_{n-1}\right)=a^{\#}\left(e^{-B t} f^{1}\right) \ldots a^{\#}\left(e^{-B t} f^{n}\right)+\mathscr{A}_{n-1}
\end{aligned}
$$


and each of these semigroups leaves all the subspaces $\mathscr{A}_{r}$ invariant. Equation (5.9) now implies that

$$
T(t)\left(W+\mathscr{A}_{n-1}\right)=a^{\#}\left(e^{C^{t}} f^{1}\right) \ldots a^{\#}\left(e^{C^{t}} f^{n}\right)+\mathscr{A}_{n-1}
$$

so

$$
\lim _{t \rightarrow \infty}\left|\left\|T(t)\left(W+\mathscr{A}_{n-1}\right) \mid\right\|=0\right.
$$

and $T(t)$ satisfies the hypotheses of Lemma 7.3.

If $X \in \mathscr{A}_{n}$ and $\varepsilon>0$ there exists $t_{0}$ such that if $t \geqq t_{0}$

$$
\left\|T_{t}(X)-\alpha 1\right\|<\varepsilon / 2
$$

where $\alpha \in \mathbb{C}$ depends on $X, t, \varepsilon$. Now $\omega_{R(\infty)}$ is an invariant state by Lemma 7.2 so

$$
\left|\omega_{R(\infty)}(X)-\alpha\right|=\left|\omega_{R(\infty)}\{T(t) X-\alpha 1\}\right|<\varepsilon / 2 .
$$

Combining the last two equations leads to

$$
\left\|T_{t}(X)-\omega_{R(\infty)}(X) 1\right\|<\varepsilon .
$$

This implies for any state $\omega$ on $\mathscr{A}$ that

$$
\left|\left(T_{t}^{*} \omega\right)(X)-\omega_{R(\infty)}(X)\right|=\left|\omega\left\{T_{t}(X)-\omega_{R(\infty)}(X) 1\right\}\right|<\varepsilon
$$

for all $t \geqq t_{0}$. Hence $T_{t}^{*} \omega$ converges to $\omega_{R(\infty)}$ in the weak* topology as $t \rightarrow \infty$.

The following special case of the above theorem will be needed in Section 8 . We introduce the operators

$$
A_{0}=\left(A+A^{*}\right) / 2, B_{0}=\left(B+B^{*}\right) / 2
$$

for notational convenience.

Theorem 7.5. Suppose that $A, A^{*}, B, B^{*}$ all lie in an abelian von Neumann algebra $\mathscr{V}$ and that $S$ is affiliated to this algebra. Then $e^{C t}$ converges strongly to zero as $t \rightarrow \infty$ if and only if $\left(A_{0}+B_{0}\right)$ is one-one, in which case

$$
R(\infty)=B_{0}\left(A_{0}+B_{0}\right)^{-1} \text {. }
$$

Proof. If $f \in \mathscr{H}$ then since the operators all commute

$$
\left\|e^{C t} f\right\|=\left\|e^{-\left(A_{0}+B_{0}\right)^{t}} f\right\|
$$

which by spectral theory and Equation (5.8) converges to zero as $t \rightarrow \infty$ if and only if 0 is not an eigenvalue of $\left(A_{0}+B_{0}\right)$. Again because all the operators commute

$$
\begin{aligned}
R(\infty) & =\int_{0}^{\infty} e^{C^{*} s}\left(B+B^{*}\right) e^{C s} d s \\
& =\int_{0}^{\infty} e^{\left(C+C^{*}\right) s}\left(B+B^{*}\right) d s \\
& =\left(B+B^{*}\right)\left(C+C^{*}\right)^{-1} \\
& =B_{0}\left(A_{0}+B_{0}\right)^{-1}
\end{aligned}
$$


Theorem 7.6. Suppose that $R, A, A^{*}, B, B^{*}$ all lie in a totally nonatomic abelian von Neumann algebra $\mathscr{V}$ and that $S$ is affiliated to this algebra. If $\left(A_{0}+B_{0}\right)$ is one-one, $0 \leqq R \leqq 1$ and

$$
R \neq B_{0}\left(A_{0}+B_{0}\right)^{-1}
$$

then the states $T_{t}^{*} \omega_{R}$ and $T_{s}^{*} \omega_{R}$ are not quasi-equivalent when $s \neq t$.

Proof. Since $R \in \mathscr{V}$, Equation (6.3) takes the form

$$
R(t)=R e^{-2\left(A_{0}+B_{0}\right) t}+B_{0}\left(A_{0}+B_{0}\right)^{-1}\left\{1-e^{-2\left(A_{0}+B_{0}\right) t}\right\} .
$$

According to [37] the states $\omega_{R(s)}$ and $\omega_{R(t)}$ are quasi-equivalent if and only if both

$$
R(s)^{1 / 2}-R(t)^{1 / 2}
$$

and

$$
\{1-R(s)\}^{1 / 2}-\{1-R(t)\}^{1 / 2}
$$

are Hilbert-Schmidt. Since $\mathscr{V}$ is totally non-atomic these imply $R(s)=R(t)$. If $s \neq t$ this in turn implies

$$
R=B_{0}\left(A_{0}+B_{0}\right)^{-1} \text {. }
$$

We suggest that this theorem implies that one cannot describe the time evolution of states in Hilbert space, or spatial, terms. One may qualitatively say that the Hilbert spaces associated to different time instants are disjoint. Thus the dynamical equations must be treated at the $C^{*}$-algebra level.

We conclude the section with an example of strong local stability.

Theorem 7.7. If $B=0$ and $e^{C t}$ converges strongly to zero as $t \rightarrow \infty$ then the Fock state $\omega_{0}$ is invariant for the dynamical semigroup $T(t)$ with evolution equation

$$
\frac{d X}{d t}=D_{S}(X)+Z_{A}(X)
$$

Moreover $\omega_{0}$ is globally stable and strongly locally stable.

Proof. Since $B=0, R(\infty)=0$ and the Fock state $\omega_{0}$ is globally stable by Theorem 7.4.

That $\omega_{0}$ is spatial with respect to $T(t)$ follows by the method of [9]. Indeed if $V_{n}$ is the space of density matrices on Fock space $\mathscr{F}$ which are supported in the subspace $\mathscr{F}_{n}$ of $\leqq n$ particles then

$$
T_{t}^{*}\left(V_{n}\right) \subseteq V_{n}
$$

for all $n$ and $t \geqq 0$.

For any operator $Y$ on $\mathscr{H}$ we define $Y^{\sim}$ to be the unbounded operator on $\mathscr{F}$ which maps each $n$-particle subspace into itself and is given there by

$$
Y \otimes 1 \otimes \ldots \otimes 1+1 \otimes Y \otimes 1 \otimes \ldots \otimes 1+\ldots+1 \otimes \ldots \otimes 1 \otimes Y
$$

The number operator $N$ therefore equals $1^{\sim}$. We denote by $P$ the projection of $\mathscr{F}$ onto the one-dimensional subspace spanned by the Fock vacuum $\Omega$. 
If the state $\psi$ on $\mathscr{A}$ is determined by an operator in $V_{n}$, and $\left\{e_{m}\right\}$ is any orthonormal basis of $\mathscr{H}$ then

$$
\begin{aligned}
\operatorname{tr}\left[\left(T_{t}^{*} \psi\right) N\right] & =\sum_{m=1}^{\infty} \operatorname{tr}\left[\left(T_{t}^{*} \psi\right) a^{*}\left(e_{m}\right) a\left(e_{m}\right)\right] \\
& =\sum_{m=1}^{\infty} \operatorname{tr}\left[\psi a^{*}\left(e^{C t} e_{m}\right) a\left(e^{C t} e_{m}\right)\right] \\
& =\psi\left\{\left(e^{C * t} e^{C t}\right)^{\sim}\right\} .
\end{aligned}
$$

Since $e^{\mathrm{C} * t} e^{\mathrm{Ct}}$ converges in the weak operator topology to 0 as $t \rightarrow \infty$

$$
\lim _{t \rightarrow \infty} \operatorname{tr}\left[\left(T_{t}^{*} \psi\right) N\right]=0 .
$$

Since

$$
0 \leqq 1-P \leqq N
$$

we have

$$
0 \leqq \operatorname{tr}\left[\left(T_{t}^{*} \psi\right)(1-P)\right] \leqq \operatorname{tr}\left[\left(T_{t}^{*} \psi\right) N\right]
$$

which together with Equation (7.5) implies that

$$
\lim _{t \rightarrow \infty}\left\langle\left(T_{t}^{*} \psi\right) \Omega, \Omega\right\rangle=1 \text {. }
$$

This in turn implies that

$$
\lim _{t \rightarrow \infty}\left\|T_{t}^{*} \psi-\omega_{0}\right\|=0 \text {. }
$$

\section{§8. A Statistical Mechanical Model}

Following the notation of Section 1, we let $\mathscr{A}$ be the CAR algebra over $\mathscr{H}=L^{2}\left(\mathbb{R}^{3}\right)$ and let $S$ be the single particle Hamiltonian $-\frac{1}{2} \Delta$. Given $f, g$ in Schwartz space $\mathscr{S}$ we consider the evolution equation

$$
\frac{d X}{d t}=D_{S}(X)+Z_{A}(X)+Y_{B}(X)
$$

on $\mathscr{A}$ where

$$
\begin{aligned}
Z_{A}(X)= & \int_{\mathbb{R}^{3}}\left\{2 a^{*}\left(f_{x}\right) \theta(X) a\left(f_{x}\right)-a^{*}\left(f_{x}\right) a\left(f_{x}\right) X\right. \\
& \left.-X a^{*}\left(f_{x}\right) a\left(f_{x}\right)\right\} d^{3} x
\end{aligned}
$$

and

$$
\begin{aligned}
Y_{B}(X)= & \int_{\mathbb{R}^{3}}\left\{2 a\left(g_{x}\right) \theta(X) a^{*}\left(g_{x}\right)-a\left(g_{x}\right) a^{*}\left(g_{x}\right) X\right. \\
& \left.-X a\left(g_{x}\right) a^{*}\left(g_{x}\right)\right\} d^{3} x .
\end{aligned}
$$

Physically $Z_{A}$ describes the spatially homogeneous absorption of particles from the system and $Y_{B}$ describes the spatially homogeneous emission of particles into the system from an external reservoir. 
By first introducing a space cut-off into the interaction terms one sees Equation (8.1) is of the form treated in Theorem 5.2 if the operators $A$ and $B$ on $\mathscr{H}$ are defined by

$$
\begin{aligned}
& A=\int_{\mathbb{R}^{3}}\left|f_{x}\right\rangle\left\langle f_{x}\right| d^{3} x \\
& B=\int_{\mathbb{R}^{3}}\left|g_{x}\right\rangle\left\langle g_{x}\right| d^{3} x .
\end{aligned}
$$

If ${ }^{\wedge}$ is the Fourier transform then

$$
\begin{aligned}
& (A \psi)^{\hat{(}(k)}=(2 \pi)^{3}|\hat{f}(k)|^{2} \hat{\psi}(k) \\
& (B \psi)^{\hat{(}(k)}=(2 \pi)^{3}|\hat{g}(k)|^{2} \hat{\psi}(k)
\end{aligned}
$$

for all $\psi \in \mathscr{H}$ and $k \in \mathbb{R}^{3}$. That is $A$ and $B$ are bounded multiplication operators in the momentum representation.

\section{Theorem 8.1. If}

$$
|\hat{f}(k)|^{2}+|\hat{g}(k)|^{2}>0
$$

for all $k \in \mathbb{R}^{3}$ then for all states $\omega$ on $\mathscr{A}$

$$
\lim _{t \rightarrow \infty} T_{t}^{*} \omega=\omega_{R(\infty)}
$$

in the weak* topology, where the operator $R(\infty)$ on $\mathscr{H}$ is defined by

$$
\{R(\infty) \psi\}^{\wedge}(k)=|\hat{g}(k)|^{2}\left\{|\hat{f}(k)|^{2}+|\hat{g}(k)|^{2}\right\}^{-1} \hat{\psi}(k)
$$

for all $\psi \in \mathscr{H}$. If $\omega$ is a spatially homogeneous quasi free state different from $\omega_{\boldsymbol{R}(\infty)}$ then $T_{t}^{*} \omega$ and $T_{s}^{*} \omega$ are not quasi-equivalent unless $s=t$. If

$$
|f(k)|^{2}>0, \quad|g(k)|^{2}>0
$$

for all $k \in \mathbb{R}^{3}$ then $\omega_{R(\infty)}$ is spatial with respect to the dynamical semigroup $T(t)$.

Proof. The formula

$$
\{(j h) \psi\}(k)=h(k) \psi(k)
$$

defines an algebra isomorphism $j$ of $L^{\infty}\left(\mathbb{R}^{3}\right)$ onto the von Neumann algebra $\mathscr{V}$ of all spatially homogeneous operators on $\mathscr{H}$. Therefore $\mathscr{V}$ is a totally non-atomic abelian von Neumann algebra. Equation (8.6) implies that the operator $\left(A_{0}+B_{0}\right)$ of Theorem 7.5 is one-one. Most of this theorem may be obtained directly from Theorems 7.4, 7.5 and 7.6.

Equation (8.8) implies that $R(\infty)$ has neither zero nor one as an eigenvalue. Therefore there exists an unbounded self-adjoint operator $H$ on $\mathscr{H}$ such that

$$
R(\infty)=\left(e^{H}+1\right)^{-1} \text {. }
$$

Therefore $\omega_{R(\infty)}$ is a KMS state with respect to the automorphism group of whose generator is $D_{H}[4$, p. $18 ; 20 ; 24]$ and hence is a separating state by $[51$, p. 69]. Therefore $\omega_{R(\infty)}$ is spatial by Theorem 4.2 . 


\section{§9. A Spatially Inhomogeneous Model}

We consider an evolution equation similar to that of the last section but with different operators $A$ and $B$. We put $A=a 1$ where $a>0$, corresponding to the physical assumption that particles are absorbed from the system at $a$ rate a which is independent of their position and momentum. We put

$$
B=\int_{\mathbb{R}^{3}} b(x)\left|g_{x}\right\rangle\left\langle g_{x}\right| d^{3} x
$$

where $g \in \mathscr{S}$ and $b$ is a non-negative bounded function representing the spatial density of an external source emitting particles into the system. If $b$ is not constant then the evolution equation

$$
\frac{d X}{d t}=D_{S}(X)+Z_{A}(X)+Y_{B}(X)
$$

with

$$
S=-\frac{1}{2} \Delta
$$

is not spatially homogeneous and interesting new phenomena can occur. Before we can use any of the theory of Section 5 we need the following lemma.

Lemma 9.1. The integral in Equation (9.1) is convergent in the weak operator topology and defines an operator $B$ with

$$
0 \leqq B \leqq\|f\|_{\infty}\|g\|_{1}^{2} 1 \text {. }
$$

Proof. As a quadratic form

$$
0 \leqq B \leqq B^{\prime} \equiv\|b\|_{\infty} \int_{\mathbb{R}^{3}}\left|g_{x}\right\rangle\left\langle g_{x}\right| d^{3} x
$$

and by Fourier analysis

$$
\left(B^{\prime} \psi\right)^{\hat{(}(k)}=(2 \pi)^{3}\|b\|_{\infty}|\hat{g}(k)|^{2} \hat{\psi}(k)
$$

for all $\psi \in \mathscr{H}$ and $k \in \mathbb{R}^{3}$, so

$$
\begin{aligned}
0 \leqq B^{\prime} & \leqq(2 \pi)^{3}\|b\|_{\infty}\|\hat{g}\|_{\infty}^{2} 1 \\
& \leqq\|b\|_{\infty}\|g\|_{1}^{2} 1 .
\end{aligned}
$$

We now denote by $T(t)$ the dynamical semigroup on $\mathscr{A}$ associated with the evolution Equation (9.2) according to Theorem 5.2.

Theorem 9.2. If the operator $R$ on $\mathscr{H}$ is defined by

$$
R=\int_{0}^{\infty} 2 e^{-2 a t} e^{(-i S-B) t} B e^{(i S-B) t} d t
$$

then $\omega_{R}$ is a temporally invariant globally stable state on $\mathscr{A}$.

Proof. Since

$$
B=B^{*} \geqq 0
$$


Equation (5.8) implies that

$$
\left\|e^{C t}\right\|=\left\|e^{(i S-B) t} e^{-a t}\right\| \leqq e^{-a t}
$$

so the conditions of Theorem 7.4 are satisfied.

The state $\omega_{R}$ is generally not spatially homogeneous. In order to investigate some of its properties we recall some well-known ideas.

The particle number distribution of any state $\omega$ on the CAR algebra $\mathscr{A}$ over $\mathscr{H}$ $=L^{2}\left(\mathbb{R}^{3}\right)$ is a non-negative countably additive measure $\mu$ on $\mathbb{R}^{3}$ such that for any Borel set $E \subseteq \mathbb{R}^{3}$

$$
\mu(E)=\sum_{n=1}^{\infty} \omega\left\{a^{*}\left(f^{n}\right) a\left(f^{n}\right)\right\}
$$

where $\left\{f^{n}\right\}$ is any orthonormal basis of the subspace $P_{E} \mathscr{H}$, and $P_{E}$ is the projection

$$
\left(P_{E} \psi\right)(x)=\left\{\begin{array}{lll}
\psi(x) & \text { if } & x \in E \\
0 & \text { if } & x \notin E .
\end{array}\right.
$$

If the operator $R$ on $\mathscr{H}$ is defined by

$$
\langle R f, g\rangle=\omega\left\{a^{*}(f) a(g)\right\}
$$

for all $f, g \in \mathscr{H}$, so that $0 \leqq R \leqq 1$, then we may alternatively write

$$
\mu(E)=\operatorname{tr}\left[R P_{E}\right]
$$

which shows that the particular orthonormal basis of $P_{E} \mathscr{H}$ chosen is not important. We say the state $\omega$ is locally finite if $\mu(E)<\infty$ for all bounded Borel sets $E$.

In the spatially homogeneous case the measure $\mu$ associated to the state $\omega_{R}$ of Theorem 9.2 can be easily determined.

Theorem 9.3. If $b(x)=1$ for all $x \in \mathbb{R}^{3}$ then $\omega_{R}$ is locally finite and its distribution $\mu$ is given by

$$
\mu(E)=\int_{\mathbb{R}^{3}} h(k) d^{3} k \int_{E} d^{3} x
$$

where

$$
h(k)=|\hat{g}(k)|^{2}\left\{(2 \pi)^{-3} a+|\hat{g}(k)|^{2}\right\}^{-1}
$$

for all $k \in \mathbb{R}^{3}$.

Proof. We first note that $h \in L^{1}\left(\mathbb{R}^{3}\right)$ since $g \in \mathscr{S}$.

A simple calculation based on Equation (9.5) shows that

$$
(R \psi) \hat{(}(k)=h(k) \hat{\psi}(k)
$$

for all $\psi \in \mathscr{H}$. The operator $R$ on $\mathscr{H}$ therefore has the positive definite continuous kernel $(2 \pi)^{-3 / 2} \hat{h}(y-x)$.

If $E$ is compact then $P_{E} R P_{E}$ is trace class by Mercer's theorem [46, p. 128] and

$$
\begin{aligned}
\operatorname{tr}\left[R P_{E}\right] & =\operatorname{tr}\left[P_{E} R P_{E}\right] \\
& =\int_{E}(2 \pi)^{-3 / 2} h(0) d^{3} x \\
& =\int_{\mathbb{R}^{3}} h(k) d^{3} k \int_{E} d^{3} x .
\end{aligned}
$$


The truth of the theorem for more general Borel sets $E$ follows by standard measure theory.

We now return to the spatially inhomogeneous case.

Theorem 9.4. For all non-negative bounded functions $b$ the state $\omega_{R}$ is locally finite. Moreover the measure $\mu$ has a bounded density with respect to Lebesgue measure.

Proof. If $E$ is a bounded Borel set then

$$
\begin{aligned}
\mu(E) & =\int_{0}^{\infty} 2 e^{-2 a t} \operatorname{tr}\left[P_{E} e^{(-i S-B) t} B e^{(i S-B) t} P_{E}\right] d t \\
& =\left.\int_{0}^{\infty} 2 e^{-2 a t}\left\|B^{1 / 2} e^{(i S-B) t} P_{E}\right\|\right|^{2} d t
\end{aligned}
$$

where $\|\cdot\| \|$ is the Hilbert-Schmidt norm. Now

$$
e^{(i S-B) t}=e^{i S t}-\int_{s=0}^{t} e^{(i S-B)(t-s)} B e^{i S s} d s
$$

so

$$
\begin{aligned}
B^{1 / 2} e^{(i S-B) t} P_{E}= & B^{1 / 2} e^{i S t} P_{E} \\
& -\int_{s=0}^{t}\left(B^{1 / 2} e^{(i S-B)(t-s)} B^{1 / 2}\right)\left(B^{1 / 2} e^{i S s} P_{E}\right) d s
\end{aligned}
$$

and

$$
\begin{aligned}
\left\|B^{1 / 2} e^{(i S-B) t} P_{E}\right\| \leqq & \left\|B^{1 / 2} e^{i S t} P_{E}\right\| \\
& +\int_{s=0}^{t}\left\|B^{1 / 2}\right\|^{2}\left\|B^{1 / 2} e^{i S s} P_{E}\right\| d s .
\end{aligned}
$$

If $B$ is defined by Equation (9.3) then

$$
\begin{aligned}
\left\|B^{1 / 2} e^{i S t} P_{E}\right\|^{2} & =\operatorname{tr}\left[P_{E} e^{-i S t} B e^{i S t} P_{E}\right] \\
& \leqq \\
& \operatorname{tr}\left[P_{E} e^{i S t} B^{\prime} e^{i S t} P_{E}\right] \\
& =\operatorname{tr}\left[P_{E} B^{\prime} P_{E}\right] \\
& =\int_{\mathbb{R}^{3}}\|b\|_{\infty}|\hat{g}(k)|^{2} d^{3} k \int_{E} d^{3} x \\
& =\|b\|_{\infty}\|g\|_{2}^{2} \int_{E} d^{3} x
\end{aligned}
$$

by Equation (9.4) and the fact that $B^{\prime}$ commutes with $e^{i s t}$. Substituting this estimate into Equation (9.8) there is a constant $\beta$ such that

$$
\left\|B^{1 / 2} e^{(i S-B) t} P_{E}\right\| \leqq \beta(1+t)\left\{\int_{E} d^{3} x\right\}^{1 / 2}
$$

for all $t \geqq 0$. Hence

$$
\begin{aligned}
\mu(E) & \leqq \int_{0}^{\infty} 2 e^{-2 a t} \beta^{2}(1+t)^{2} d t \int_{E} d^{3} x \\
& =\gamma \int_{E} d^{3} x .
\end{aligned}
$$


By the Radon-Nikodym theorem the density of $\mu$ with respect to Lebesgue measure is bounded by $\gamma$.

We specialise further to the case where the emission of particles into the system from the reservoir occurs only in the region

$$
\left.\mathbb{R}_{+}^{3}=(u, v, w) \in \mathbb{R}^{3}: u>0\right\} .
$$

This is achieved by putting

$$
b(x)=\left\{\begin{array}{lll}
1 & \text { if } & x \in \mathbb{R}_{+}^{3} \\
0 & \text { if } & x \notin \mathbb{R}_{+}^{3} .
\end{array}\right.
$$

For notational convenience we shall write $u$ below for the element $(u, 0,0)$ of $\mathbb{R}^{3}$.

Physically one would expect that the state $\omega_{R}$ shows a transition as one passes from large negative values of $u$ to large positive values. This is made precise in the following theorem.

\section{Theorem 9.5. The limit states}

$$
\omega^{ \pm}=\lim _{u \rightarrow \pm \infty} \alpha_{u}^{*} \omega_{R}
$$

exist in the weak $*$ topology and are spatially homogeneous quasi-free states of $\mathscr{A}$. The state $\omega^{-}$is the Fock state while $\omega^{+}$is the quasi-free state associated to $R^{+}$, where

$$
\left(R^{+} \psi\right)^{\hat{n}}(k)=h(k) \hat{\psi}(k)
$$

for all $\psi \in \mathscr{H}$, and $h$ is defined by Equation (9.7).

Proof. Since

$$
\begin{aligned}
& \left(\alpha_{u}^{*} \omega_{R}\right)\left\{a^{*}\left(f^{1}\right) \ldots a^{*}\left(f^{m}\right) a\left(g^{n}\right) \ldots a\left(g^{1}\right)\right\} \\
& =\omega_{R}\left\{a^{*}\left(U_{u} f^{1}\right) \ldots a\left(U_{u} g^{1}\right)\right\} \\
& =\delta_{m n} \operatorname{det}\left\{\left\langle R\left(U_{n} f^{i}\right),\left(U_{n} g^{j}\right)\right\rangle\right\} \\
& \omega_{U_{u}^{*} R U_{u}}\left\{a^{*}\left(f^{1}\right) \ldots a\left(g^{1}\right)\right\}
\end{aligned}
$$

for all normal ordered Wick monomials, we see that

$$
\alpha_{u}^{*} \omega_{R}=\omega_{U_{u}^{*} R U_{u}} .
$$

By Lemma 6.1 we have to prove that

$$
R_{u}=U_{u}^{*} R U_{u}
$$

converge in the weak operator topology to $R^{+}$(resp. $R^{-}$) as $u$ tends to $+\infty$ (resp. $-\infty)$.

By Equation (9.5) and the spatial homogeneity of $S$

$$
R_{u}=\int_{0}^{\infty} 2 e^{-2 a t} e^{\left(-i S-B_{u}\right) t} B_{u} e^{\left(i S-B_{u}\right) t} d t
$$

where

$$
B_{u} \equiv U_{u}^{*} B U_{u}=\int_{\mathbb{R}^{3}} b(x+(u, 0,0))\left|g_{x}\right\rangle\left\langle g_{x}\right| d^{3} x .
$$


It is clear that as $u \rightarrow+\infty, B_{u}$ converges in the weak operator topology to

$$
B^{+}=\int_{\mathbb{R}^{3}}\left|g_{x}\right\rangle\left\langle g_{x}\right| d^{3} x .
$$

Since also $u \leqq v$ implies $B_{u} \leqq B_{v}, B_{u}$ actually converges to $B^{+}$in the strong operator topology. This implies by [31, p. 502] that

$$
\lim _{u \rightarrow+\infty} e^{\left(i S-B_{u}\right) t}=e^{\left(i S-B^{+}\right) t}
$$

in the strong operator topology for all $t \geqq 0$. It follows by the dominated convergence theorem that $R_{u}$ converges in the weak operator topology to

$$
R^{+}=\int_{0}^{\infty} 2 e^{-2 a t} e^{\left(-i S-B^{+}\right) t} B^{+} e^{\left(i S-B^{+}\right) t} d t
$$

The required expression for $R^{+}$may be obtained as in Theorem 9.3.

The case $u \rightarrow-\infty$ is done similarly, the limit operator $R^{-}$being equal to 0 , which characterises the Fock state.

\section{References}

1. Alfsen,E.M.: Borel structure on a metrizable Choquet simplex and on its extreme boundary. Math. Scand. 19, 161-171 (1966)

2. Alfsen,E.M.: Compact convex sets and boundary integrals. Berlin-Heidelberg-New York: Springer 1971

3. Araki,H., Sewell,G.L.: KMS conditions and local thermodynamic stability of quantum lattice systems. Commun. math. Phys. 52, 103-109 (1977)

4. Araki,H., Wyss, W.: Representations of canonical anticommutation relations. Helv. Phys. Acta 37, 136-159 (1964)

5. Balslev,E., Verbeure,A.: States on Clifford algebras. Commun. math. Phys. 7, 55-76 (1968)

6. Bratteli, O., Robinson,D.W.: Unbounded derivations in operator algebras. Commun. math. Phys. 42, 253-268 (1975)

7. Chernoff,P.R.: Note on product formulas for operator semigroups. J. Funct. Anal. 2, 238-242 (1968)

8. Davies, E.B.: Markovian master equations. Commun. math. Phys. 39, 91-110 (1974)

9. Davies,E.B.: Quantum dynamical semigroups and the neutron diffusion equation. Rept. math. Phys. 11, 169-188 (1977)

10. Davies, E. B.: First and second quantised neutron diffusion equations. Commun. math. Phys. 52, $111-126(1977)$

11. Davies, E.B.: Dilations of completely positive maps. To appear

12. Davies,E.B.: Quantum theory of open systems. New York: Academic Press 1976

13. Davies, E. B., Eckmann,J.-P.: Time decay for fermion systems with persistent vacuum. Helv. Phys. Acta 48, 731-742 (1975)

14. Dell'Antonio, G.F.: Structure of the algebras of some free systems. Commun. math. Phys. 9, 81-117 (1968)

15. Dixmier,J. : Les algèbres d'operateurs dans l'espace hilbertien. 2nd ed. Paris: Gauthier-Villars 1969

16. Doplicher,S., Kadison,R.V., Kastler,D., Robinson,D.W.: Asymptotically abelian systems. Commun. math. Phys. 6, 101-120 (1967)

17. Doplicher,S., Powers, R.T.: On the simplicity of the even CAR algebra and free field models. Commun. math. Phys. 7, 77-79 (1968)

18. Dubin,D.: Solvable models in algebraic statistical mechanics. Oxford: Univ. Press 1974

19. Emch,G.G.: Algebraic methods in statistical mechanics and quantum field theory. New York: Wiley-Interscience 1972

20. Evans, D.E. : Positive linear maps on operator algebras. Commun. math. Phys. 48, 15-22 (1976) 
21. Evans,D.E., Lewis,J.T.: Completely positive maps on some $C^{*}$-algebras. Preprint

22. Evans, D.E., Lewis,J.T.: Dilations of dynamical semigroups. Commun. math. Phys. 50, 219-227 (1976)

23. Gorini, V., Kossakowki, A.: N-level systems in contact with a singular reservoir. J. Math. Phys. 17, $1298-1305$ (1976)

24. Haag, R., Hugenholtz,N.M., Winnink,M.: On the equilibrium states in quantum statistical mechanics. Commun. math. Phys. 5, 215-236 (1967)

25. Haag, R., Kadison, R.V., Kastler,D.: Nets of $C^{*}$-algebras and classification of states. Commun. math. Phys. 16, 81-104 (1970)

26. Haag, R., Kastler,D., Trych-Pohlmeyer,E.B.: Stability and equilibrium states. Commun. math. Phys. 38, 173-193 (1974)

27. Haake, F.: Statistical treatment of open systems by generalised master equations. Springer tracts in modern physics, Vol. 66. Berlin-Heidelberg-New York: Springer 1973

28. Hoegh-Krohn, R. : Asymptotic fields in some models of quantum field theory II. J. Math. Phys. 10, 639-643 (1969)

29. Hugenholtz,N.M., Kadison, R.V.: Automorphisms and quasi-free states on the CAR algebra. Commun. math. Phys. 43, 181-197 (1975)

30. Kadison, R. V.: Transformations of states in operator theory and dynamics. Topology 3 (Suppl. 2), 177-198 (1965)

31. Kato, T.: Perturbation theory for linear operators. Berlin-Heidelberg-New York: Springer 1966

32. Kraus, K.: General state changes in quantum theory. Ann. Phys. 64, 311-335 (1971)

33. Lindblad,G.: On the generators of quantum dynamical semigroups. Commun. math. Phys. $\mathbf{4 8}$, 119-130 (1976)

34. Manuceau,J., Rocca,F., Testard,D. : On the product form of quasi-free states. Commun. math. Phys. 12, 43-57 (1969)

35. Palmer, P.F.: The singular coupling and weak coupling limits. J. Math. Phys. 18, 527-529 (1977)

36. Powers, R.T., Sakai, S.: Existence of ground states and KMS states for approximately inner dynamics. Commun. math. Phys. 39, 273-288 (1975)

37. Powers, R.T., Størmer,E.: Free states of the canonical anticommutation relations. Commun. math. Phys. 16, 1-33 (1970)

38. Pulè,J.: The Bloch equations. Commun. math. Phys. 38, 241-256 (1974)

39. Reed,M., Simon, B. : Methods of modern mathematical physics, Vol. 2. New York : Academic Press 1975

40. Robinson,D.W.: Statistical mechanics of quantum spin systems II. Commun. math. Phys. 7, 21-32 (1970)

41. Robinson,D.W.: Return to equilibrium. Commun. math. Phys. 31, 171-189 (1973)

42. Ruelle,D.: Statistical mechanics. New York: W. A.Benjamin Inc. 1969

43. Schatten, R.: A theory of cross-spaces. Princeton: Univ. Press 1950

44. Schrader, R., Uhlenbrock,D. A. : Markov structures on Clifford algebras. J. Funct. Anal. 18, 369$413(1975)$

45. Shale,D., Stinespring,W.F.: States of the Clifford algebra. Ann. Math. 80, 365-381 (1964)

46. Smithies, F.: Integral equations. Cambridge: Univ. Press 1962

47. Stinespring,W. F.: Positive functions on $C^{*}$-algebras. Proc. Amer. Math. Soc. 6, 211-216 (1955)

48. Størmer,E.: The even CAR algebra. Commun. math. Phys. 16, 136-137 (1970)

49. Streater, R.F.: On certain non-relativistic quantised fields. Commun. math. Phys. 7, 93-98 (1968)

50. Streater,R.F., Wilde,I.F.: The time evolution of quantised fields with bounded quasi-local interaction density. Commun. math. Phys. 17, 21-32 (1970)

51. Takesaki,M.: Tomita's theory of modular Hilbert algebras and its applications. Lecture notes in math. Vol. 128. Berlin-Heidelberg-New York: Springer 1970

52. Yosida,K.: Functional analysis. Berlin-Heidelberg-New York : Springer 1965

Communicated by J.L.Lebowitz 